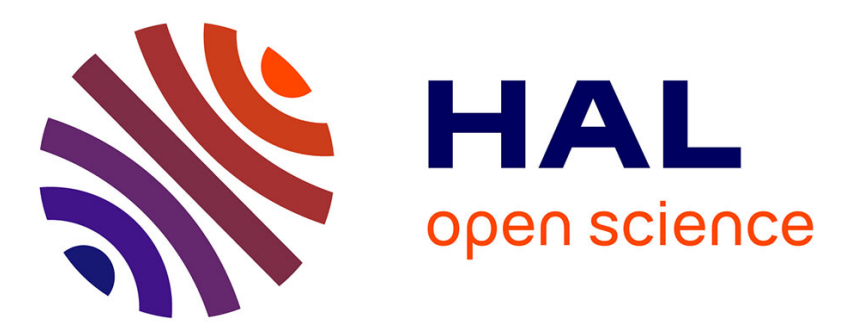

\title{
On compressibility assumptions in aeroacoustic integrals: a numerical study with subsonic mixing layers
}

Florent Margnat, Xavier Gloerfelt

\section{To cite this version:}

Florent Margnat, Xavier Gloerfelt. On compressibility assumptions in aeroacoustic integrals: a numerical study with subsonic mixing layers. Journal of the Acoustical Society of America, 2014, 135 (6), pp.3252. 10.1121/1.4875561 . hal-01069755

\section{HAL Id: hal-01069755 https://hal.science/hal-01069755}

Submitted on 29 Sep 2014

HAL is a multi-disciplinary open access archive for the deposit and dissemination of scientific research documents, whether they are published or not. The documents may come from teaching and research institutions in France or abroad, or from public or private research centers.
L'archive ouverte pluridisciplinaire HAL, est destinée au dépôt et à la diffusion de documents scientifiques de niveau recherche, publiés ou non, émanant des établissements d'enseignement et de recherche français ou étrangers, des laboratoires publics ou privés. 


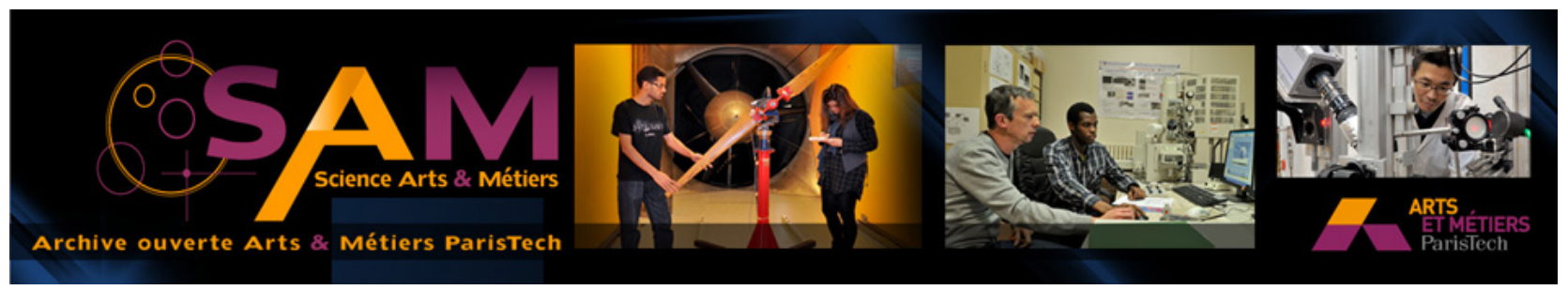

Science Arts \& Métiers (SAM)

is an open access repository that collects the work of Arts et Métiers ParisTech researchers and makes it freely available over the web where possible.

This is an author-deposited version published in: http://sam.ensam.eu

Handle ID: .http://hdl.handle.net/10985/8641

\section{To cite this version :}

Florent MARGNAT, Xavier GLOERFELT - On compressibility assumptions in aeroacoustic integrals: a numerical study with subsonic mixing layers - The Journal of the Acoustical Society of America - Vol. 135, nº, p.3252 - 2014 


\title{
On compressibility assumptions in aeroacoustic integrals: a numerical study within subsonic mixing layers
}

\author{
Florent Margnat ${ }^{\mathrm{a})}$ \\ Institute PPRIME, Department of Fluid Flow, Heat Trans- \\ fer and Combustion, Université de Poitiers - ENSMA - CNRS \\ Building B17 - 6 rue Marcel Doré - TSA 41105 \\ 86073 POITIERS CEDEX 9 - France \\ Xavier Gloerfelt \\ Arts et Metiers ParisTech, DynFluid Lab, 151 boulevard de l'Hopital, 75013 PARIS, France
}

(Dated: March 24, 2014)

\begin{abstract}
Two assumptions commonly made in predictions based on Lighthill's formalism are investigated: a constant density in the quadrupole expression, and the evaluation of the source quantity from incompressible simulations. Numerical predictions of the acoustic field are conducted in the case of a subsonic spatially evolving two-dimensional mixing layer at $R e=400$. Published results of the direct noise computation (DNC) of the flow are use as reference and input for hybrid approaches before the assumptions on density are progressively introduced. Divergence free velocity fields are obtained from an incompressible simulation of the same flow case, exhibiting the same hydrodynamic field as the DNC. Fair comparisons of the hybrid predictions with the reference acoustic field valid both assumptions in the source region for the tested values of the Mach number. However, in the observer region, the inclusion of flow effects in the Lighthill source term is not preserved, which is illustrated through a comparison with the Kirchhoff wave-extrapolation formalism, and with the use of a convected Green function in the integration process.
\end{abstract}

PACS numbers: 43.28.Ra, 43.20.Wd

\section{INTRODUCTION}

The overall problem area addressed here is that of estimating the noise radiated by unsteady flows. We aim at improving the class of methods in two steps, usually described as hybrid, which use experimentally or numerically generated flow data as an input for solutions of wave propagation equations, in particular those solutions written with an integral formalism such as Lighthill's analogy and the Kirchhoff wave extrapolation method. Because the two-step approach allows cost reduction in the estimation of the radiated field, hybrid methods are frequently chosen for the prediction of flow generated noise in many applications such as jet, high-lift devices and landing-gear on planes, car mirror and window vortex, fans, propellers, etc. That prediction consists in extracting among the unsteady motions in the flow those able to excite outward propagating sound waves. Consequently, once it is obtained, the analysis of the acoustic field and its correlation to the flow events can be conducted in order to identify the noisy vortical motion. In that sense, hybrid methods may also help the physical understanding of aeroacoustic issues.

Any analogy approach relies on the explicit knowledge of the source quantity that excites the wave operator. Since Lighthill's initial derivation ${ }^{1}$ of an inhomogeneous wave equation from the equations of motion, with a source term defined by the flow quantities, a constant

\footnotetext{
a) Electronic address: florent.margnat@univ-poitiers.fr
}

research effort has been devoted to improve its potential, from theoretical works on the source term and surrounding flow effect modelling, to numerical works increasing quickness and accuracy of the solution. In particular, Crow $^{2}$ investigated analytically the distinct role of the compressibility in the source term and in the wave operator. By the method of matched asymptotic expansion, he concluded that, for low Mach number and compact source region, Lighthill's solution for the density is adequate if the latter is assumed constant in the quadrupole.

Because Lighthill's equation is an exact rearrangement of the flow equations, it contains flow effects on acoustics such as wave convection and refraction. The left-hand side being the wave operator without flow, those effects are included in the source side. Consequently, the source term thus obtained is not the true $^{3}$ acoustic source, because it accounts for other phenomena in addition to acoustic energy production. Questing for such a reduced source expression, Lilley ${ }^{4}$ and Ribner ${ }^{5}$ derived an analogy equation for jet noise modelling, through the specification of unidirectional, transversely sheared, mean flow in the Lighthill source term, so that its solution displays sound refraction by velocity gradients. This was numerically applied to a mixing layer by Colonius et $a l^{6}$. Recently, Suzuki \& Lele ${ }^{7}$ derived approximate Green functions for a source in a mixing layer. It should be the appropriate propagator to associate, in the convolution integral, with a source expression that would be purified from convection and refraction effects.

The full knowledge of the source term with fine space and time resolutions brought by the numerical simulations allows to check the validity of assumptions 
made in theoretical or experimental studies. Moreover, building reduced-order models of the flow may reduce the computational cost of closed-loop control systems. In that context, Samanta et $a l^{8}$ studied the sensitivity of several analogy formulations to errors intentionnally introduced into the source quantity, namely removing the highest modes from the decomposition or perturbating the most energetic ones.

The present contribution builds on these efforts to characterise the content of the Lighthill source term, in order to provide background for current source term evaluation in analogies. The assumptions regarding density are investigated numerically, through the association of a constant density with compressible velocity fields, as well as the evaluation of the quadrupoles from fully incompressible data. We also analyse how those assumptions are sensitive to Mach number variations and how they affect the inclusion of flow effects on acoustics in Lighthill's formalism. For the latter, the convected wave equation and the Kirchhoff formalism are used as reference points.

Numerical evaluations of aeroacoustic integrals are thus conducted in the frequency domain in the case of a subsonic spatially evolving two-dimensional mixing layer at $R e=400$. Such flow is free from surface effect such as reflexion or diffraction, thus focusing our analysis on the original source term. Moreover, a two-dimensional configuration allows the integration of many source quantities over many volume extents with an affordable computational effort. The integrands are read from databases generated by the numerical solution of the Navier-Stokes equations. A direct noise computation (DNC) provides the compressible velocity, pressure and density fields, and the reference result for the acoustic field at the same time. In addition, an incompressible simulation provides the source quantity using divergence free velocity field. To the best of the authors' knowledge, the resolution of Lighthill's equation with source data from a DNC and an incompressible simulation of the same flow was not conducted before.

The paper is organised as follows. In section II, Lighthill's and Kirchhoff's formalisms are recalled in the Fourier space, for either a convected wave equation or a propagation in a medium at rest. In section III, the mixing-layer parameters are presented along with the numerical methods applied for the flow simulations and integral evaluations. The coherence between the several prediction strategies is checked in section IV, and the inclusion of the convection effect in the Lighthill source term is illustrated. The influence of compressibility in the evaluation of the quadrupoles is then adressed in section V. Eventually, the main results of the paper are summarised and further discussed in section VI.

\section{MATHEMATICAL FORMULATION}

\section{A. Kirchhoff's formalism}

For a moving medium, the acoustic pressure $p^{\prime}$ at any time $t$ and location $\mathbf{x}$ in a volume $V$ is related to the distribution $Q$ of sources within $V$ and the distribution of the pressure and its derivative on the boundary of $V$, noted $\Sigma$, by the generalised Green formula ${ }^{9}$. For a 2-D configuration, with $\mathbf{U}^{\infty}=\left(U^{\infty}, 0\right)$ in the observer domain, it can be written as

$$
\begin{array}{r}
p^{\prime}(\mathbf{x}, t)=\int_{-\infty}^{\infty} \iint_{V} Q(\mathbf{y}, \tau) \tilde{G}(\mathbf{x}, t \mid \mathbf{y}, \tau) \mathrm{d} \mathbf{y} \mathrm{d} \tau \\
+\int_{-\infty}^{\infty} \int_{\Sigma}\left\{\tilde{G} \frac{\partial p^{\prime}}{\partial y_{i}}-p^{\prime} \frac{\partial \tilde{G}}{\partial y_{i}}\right\} n_{i} \mathrm{~d} \Sigma(\mathbf{y}) \mathrm{d} \tau \\
+\frac{U^{\infty}}{c_{0}^{2}} \int_{-\infty}^{\infty} \int_{\Sigma}\left\{p^{\prime} \frac{\mathrm{D}_{\infty} \tilde{G}}{\mathrm{D} \tau}-\tilde{G} \frac{\mathrm{D}_{\infty} p^{\prime}}{\mathrm{D} \tau}\right\} n_{1} \mathrm{~d} \Sigma(\mathbf{y}) \mathrm{d} \tau
\end{array}
$$

where $\mathbf{n}=\left(n_{1}, n_{2}\right)$ is the unit normal vector pointing inward the observer domain $V, c_{0}$ is the sound speed of the ambient fluid, $\mathrm{D}_{\infty} / \mathrm{D} t=\partial / \partial t+U_{i}^{\infty} \partial / \partial x_{i}$, and $\tilde{G}$ is the time-domain Green function solution to the uniformly moving medium wave equation. By taking the following Fourier transform:

$$
F[f(\mathbf{x}, t)]=f(\mathbf{x}, \omega)=\int_{-\infty}^{\infty} f(\mathbf{x}, t) e^{-i \omega t} \mathrm{~d} t
$$

where $\omega$ is the angular frequency and $i^{2}=-1$, formula (1) reduces to the form

$$
\begin{array}{r}
p^{\prime}(\mathbf{x}, \omega)=\iint_{V} Q(\mathbf{y}, \omega) G(\mathbf{x} \mid \mathbf{y}, \omega) \mathrm{d} \mathbf{y} \\
+\iint_{\Sigma}\left\{G(\mathbf{x} \mid \mathbf{y}, \omega) \frac{\partial p^{\prime}(\mathbf{y}, \omega)}{\partial y_{i}}\right. \\
\left.-p^{\prime}(\mathbf{y}, \omega) \frac{\partial G(\mathbf{x} \mid \mathbf{y}, \omega)}{\partial y_{i}}\right\}\left(n_{i}-M_{\infty}^{2} n_{1}\right) \mathrm{d} \Sigma(\mathbf{y}) \\
+\frac{2 i \omega M_{\infty}}{c_{0}} \int_{\Sigma} p^{\prime}(\mathbf{y}, \omega) G(\mathbf{x} \mid \mathbf{y}, \omega) \mathrm{d} \Sigma(\mathbf{y})
\end{array}
$$

where the time factor $\exp (-i \omega t)$ has been omitted. The Green function for the propagation in a uniform flow is given in the 2-D frequency domain by ${ }^{10-12}$ :

$$
G_{c}(\mathbf{x} \mid \mathbf{y}, \omega)=\frac{i}{4 \beta} \exp \left(\frac{i M_{\infty} k r_{1}}{\beta^{2}}\right) H_{0}^{(2)}\left(\frac{k r_{\beta}}{\beta^{2}}\right)
$$

where $\mathbf{y}=\left(y_{1}, y_{2}\right)$ and $\mathbf{x}=\left(x_{1}, x_{2}\right)$ are the source and observer locations respectively, and $r_{i}=x_{i}-y_{i}$. $H_{\nu}^{(m)}$ is the Hankel function of order $\nu$ and kind $m$, $k=\omega / c_{0}, \beta^{2}=1-M_{\infty}^{2}$ is the Prandtl-Glauert factor, and $r_{\beta}=\sqrt{\left(x_{1}-y_{1}\right)^{2}+\beta^{2}\left(x_{2}-y_{2}\right)^{2}}$. The Green function for the uniform medium at rest, and the related pressure formula, are recovered when $M_{\infty}$ is set to 0 .

In our configuration sketched in figure 1, the extrapolation of the acoustic waves from the control surface $\Sigma=\left(y_{2}=y_{2}^{s}\right) \cup\left(y_{2}=-y_{2}^{s}\right)$ is obtained by the following formula:

$$
\begin{array}{r}
p^{\prime}(\mathbf{x}, \omega)=\frac{n_{2} i}{4 \beta} \int_{\Sigma}\left[\frac{\partial p^{\prime}}{\partial y_{2}} H_{0}^{(2)}\left(\frac{k r_{\beta}}{\beta^{2}}\right)\right. \\
\left.-p^{\prime} \frac{k r_{\beta}}{\beta^{2}} H_{1}^{(2)}\left(\frac{k r_{\beta}}{\beta^{2}}\right)\right] \exp \left(\frac{i M_{\infty} k r_{1}}{\beta^{2}}\right) \mathrm{d} y_{1}
\end{array}
$$

Kirchhoff integral (5) theoretically predicts the acoustic pressure field provided that no source phenomenon is left 


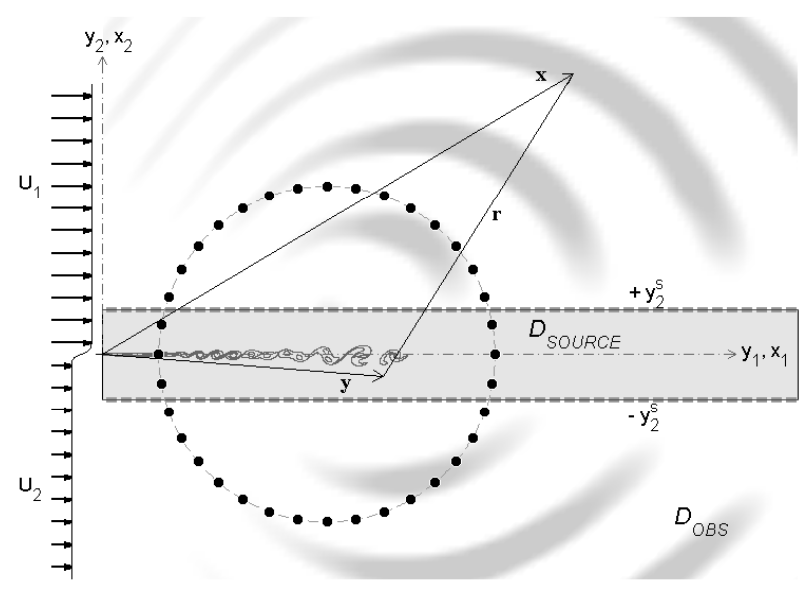

FIG. 1. Configuration and notations for the application of aeroacoustic integrals to the mixing layer flow.

inside $V$, that the pressure distribution on $\Sigma$ contains the acoustic information generated by the sources outside $V$, and convection is the only flow effect acting inside $V$.

\section{B. Lighthill's formalism}

Lighthill's equation combines the mass and momentum conservation equation to form an inhomogeneous wave equation for the density. Consequently, for an observer located in the acoustic (isentropic) region, the solution for the 2-D acoustic pressure field is given, in the frequency domain, by:

$$
p^{\prime}(\mathbf{x}, \omega)=\frac{i}{4} \iint_{D} S(\mathbf{y}, \omega) H_{0}^{(2)}(k r) \mathrm{d} \mathbf{y}
$$

where $D$ is the source region and $r=\|\mathbf{x}-\mathbf{y}\|$, for a propagation in an unbounded medium at rest. In (6), $S$ is the Lighthill source term, defined by:

$$
S=\frac{-\partial^{2} T_{i j}}{\partial y_{i} \partial y_{j}}=\frac{-\partial^{2}}{\partial y_{i} \partial y_{j}}\left[\rho u_{i} u_{j}+\left(p^{\prime}-c_{0}^{2} \rho^{\prime}\right) \delta_{i j}+\tau_{i j}\right]
$$

where $u_{i}$ are the components of the velocity field $\mathbf{u}=$ $(u, v), \rho^{\prime}$ is the density fluctuation from its value in the medium at rest and $\tau_{i j}$ stands for the components of the viscous stress tensor $\tau$.

Expression (6) can be transformed taking the space derivatives over the Green function instead of the source term, leading to:

$$
\begin{aligned}
p^{\prime}(\mathbf{x}, \omega) & =\frac{-i}{4} \iint_{D}\left[k^{2} \frac{r_{i} r_{j}}{r^{2}} H_{2}^{(2)}(k r)\right. \\
& \left.-k \frac{\delta i j}{r} H_{1}^{(2)}(k r)\right] T_{i j}(\mathbf{y}, \omega) \mathrm{d} \mathbf{y}
\end{aligned}
$$

In the case of a propagation in a uniform flow at $\mathbf{U}^{\infty}=$ $\left(U_{1}^{\infty}, U_{2}^{\infty}\right)$, the derivation of a convected wave equation ${ }^{11}$ yields:

$$
p^{\prime}(\mathbf{x}, \omega)=\iint_{D} S_{c}(\mathbf{y}, \omega) G_{c}(\mathbf{x} \mid \mathbf{y}, \omega) \mathrm{d} \mathbf{y}
$$

where

$$
\begin{aligned}
S_{c} & =\frac{\partial^{2} T_{i j}^{c}}{\partial y_{i} \partial y_{j}} \\
& =\frac{\partial^{2}\left[\rho\left(u_{i}-U_{i}^{\infty}\right)\left(u_{j}-U_{j}^{\infty}\right)+\left(p^{\prime}-c_{0}^{2} \rho^{\prime}\right) \delta_{i j}+\tau_{i j}\right]}{\partial y_{i} \partial y_{j}}
\end{aligned}
$$

Note that it is not harmless for the physical interpretation that the source expression depends on the propagation medium governing equation. It is indeed an intrinsic consequence of the analogy formalism, as explained by several authors ${ }^{3,8,13}$.

Similarly to the non-convected case, the space derivatives can be analytically transferred to the Green function instead of numerically estimated on the source term. The second-order derivatives of the convected Green function (4) are given in ${ }^{11}$.

\section{FLOW CONFIGURATION AND NUMERICAL TOOLS}

\section{A. Reference flow and source data}

As a reference, the acoustic field radiated by a spatially evolving subsonic mixing layer is obtained by directly solving the compressible Navier-Stokes equations on a spatial domain containing both the mixing region and the acoustic far field. The flow configuration and solver are the same as in ref. ${ }^{14}$.

The frame origin is at the inflow on the centerline, and the mean inflow velocity profile, illustrated in figure 1, has a hyperbolic tangent shape

$$
\bar{u}\left(0, y_{2}\right)=U_{c}+\frac{\Delta U}{2} \tanh \left(\frac{2 y_{2}}{\delta_{\omega}}\right)
$$

where $U_{c}=\frac{U_{1}+U_{2}}{2}$ and $\Delta U=U_{1}-U_{2}$, noting $U_{1}$ and $U_{2}$ the high- and low-speed flow respectively. The vorticity thickness, which varies in the streamwise direction, is defined as

$$
\delta_{\omega}\left(y_{1}\right)=\frac{\Delta U}{\max _{y_{2}}\left(\frac{\partial \bar{u}}{\partial y_{2}}\right)}
$$

Its value at the inflow is noted $\delta_{\omega}$ and is taken as the reference length throughout the paper.

Two subsonic isothermal cases with $U_{1}=2 U_{2}$ are considered here, with different value of $M=\Delta U / c_{0}$ : 0.25 and 0.40 . The Reynolds number is defined by $R e=\rho_{0} \delta_{\omega} \Delta U / \mu$, where $\rho_{0}$ is the density of the surrounding fluid (set to unity in those adimensionalized equations) and $\mu$ is the dynamic viscosity, and its value is fixed at $R e=400$. In order to obtain a periodic and spatially fixed vortex pairing phenomenon, the flow is pertubed just downstream of the inlet, at the frequency $f_{0}=0.132 U_{c} /\left(\delta_{\omega, f}\right)$ (where $\delta_{\omega, f}=2 \delta_{\omega}$ is the local vorticity thickness at $x \approx 80$ ), and its first subharmonic $f_{0} / 2$, using a divergence-free forcing function with low amplitude, as presented in ref. ${ }^{14}$.

The space derivatives are evaluated using sixth-order compact finite difference schemes, while a third-order 
Runge-Kutta scheme provides the time marching. Finally, the boundary conditions are written using a characteristic formulation, and terms are set inflow and outflow. The computational domain lays over $800 \delta_{\omega}$ in both directions. The grid size is $(2071 \times 785)$. It is stretched from the centerline in $y_{2}$ direction. In $y_{1}$ direction, it is almost constant below $400 \delta_{\omega}$, and streched after, combined with a dissipation function to create the sponge zone. The flow solver and numerical parameters are described in details in ref. ${ }^{14}$.

These simulations provide both the reference acoustic field and the input data for the Kirchhoff solution (5) and the computation of the Lighthill source quantities (7), (10), (15) and (16).

\section{B. Hybrid method implementations}

Thanks to the periodic nature of the flow, the computation of the aeroacoustic integrals can be conducted in the frequency domain thus avoiding the integration over the whole history of the time series that is implied by $2 \mathrm{D}$ Green function in the time domain . Standard Fast Fourrier Transform routines are applied to the source time series. The latter are recorded about 500 times a period from the direct computation. The Hankel functions are computed using Amos library ${ }^{15}$. A mid-panel quadrature is implemented for the integration of the Lighthill source term, while the Kirchhoff surface integral are evaluated with a trapezoidal rule. Streamwise, the integration domain extends over the whole DNC domain. The observer grid covers the DNC domain and is uniform with 150 points in each direction, leading to $\Delta x f_{0} / c_{0} \approx 8$. For the formulations based on a convected wave equation, one sets $\mathbf{U}^{\infty}=\left(U_{1}, 0\right)$ and $M_{\infty}=U_{1} / c_{0}$ for the high-speed flow, and $\mathbf{U}^{\infty}=\left(U_{2}, 0\right)$ and $M_{\infty}=U_{2} / c_{0}$ for the low-speed flow.

The truncation of the source domain, typically at the outflow, can be treated either by a dissipation region, a spatial weighting, or the addition of a residual term based on the Reynolds transport theorem ${ }^{16}$ accounting for the missed region. Here, the two first techniques are used. Indeed, the dissipation function used in the direct computation prevents the paired vortices to generate spurious acoustic waves as they pass outflow, but their damping is not sufficient to avoid a strong contribution to the integral. Thus a weighting function based on a Tukey windowing is applied to the source term (7). With respect to the spatial top-hat window which is de facto applied when integrating (6) over the computational domain, such weighting function reduces the spectral leakage ${ }^{17}$, that is spatial spurious noise directly translated into temporal noise through the dispersion relation. The source term expression in the convected form (10) appeared more sensitive to truncation effects, that is why a weighting of the following Gaussian form is applied:

$$
W\left(y_{1}\right)=\exp \left(-\left[\frac{y_{1}-y_{1}^{0}}{\sigma\left(y_{1}^{L}-y_{1}^{0}\right)}\right]^{4}\right)
$$

where $y_{1}^{0}$ is the position before which $W$ is set to $1 ., y_{1}^{L}$ and $\sigma$ being adjusted by the user for a given computational configuration in order that no radiation is emitted from the outflow. Those weighting functions have a streamwise extent from $500 \delta_{\omega}$ to the outflow boundary.

The numerical singularity in the Hankel function, appearing when the observer is located inside the integration domain, can be treated by removing a small disk around it when computing the integral ${ }^{18}$, or by setting the observer grid in a staggered way with respect to the source grid. That latter solution suits better when the spatial derivatives are evaluated on the Green function itself.

\section{COHERENCE OF THE PREDICTION STRATEGIES}

\section{A. Validation of the hybrid methods}

The present bi-harmonically excited mixing layer experiences a periodic vortex-pairing phenomenon, which occurs around $y_{1}=200 \delta_{\omega}$, and which generates acoustic waves at that pairing frequency. The resulting instantaneous acoustic pressure field, obtained by the DNC, is plotted in figure 2a). Both Kirchhoff's (5) and Lighthill's (9) formalisms, based on a convected Green's function and fed by source data from the DNC, accurately predict that acoustic field, as visible in figure $2 \mathrm{~b}$ ) and 2c). The comparison is fair with the field directly computed solving the compressible Navier-Stokes equations plotted in figure 2a). In particular, the wavefront pattern, with a maximum radiation around $\theta=50^{\circ}$ on both layer sides, is well recovered (here and henceforth, $\theta$ is the angle counted counterclockwise from the downstream direction and centered on the pairing location).

In figure $2 \mathrm{~d}$ ), the acoustic field is plotted resulting from the numerical evaluation of Lighthill's solution (6) with the Green function for an observer medium at rest integrated over the full DNC domain. The fair comparaison to the field directly computed illustrates well that the Lighthill analogy accounts for the convection effects, yet this is the case here because the source data come from the DNC itself and the integration domain extends over the whole observer region. The integration of a source term estimated from a compressible solver, over a domain including the observer, is then found equivalent to the application of a convected Green function to a localised source region, as firstly described by Goldstein ${ }^{9}$ and numerically illustrated by Bogey et $a l^{19}$. As shown in section $\mathrm{V}$, that does not hold any more when a constant density is assumed in the quadrupole.

The mean square value of the fluctuating pressure is plotted in figure $3 \mathrm{a}$ ) on a circle at $r=150 \delta_{\omega}$ away from the apparent source location $\left(x_{1}=200 \delta_{\omega}, x_{2}=0\right)$. This quantity approaches the acoustic intensity in the far field, and provides here a more quantitative comparison between the four methods. They agree well for all radiation angles. In particular, the level fall for increasing $|\theta|$ is perfectly reproduced by the hybrid methods. For $|\theta|$ higher than $90^{\circ}$, some directivity lobes are visible, at very low levels. The method using the integration of the 
a)

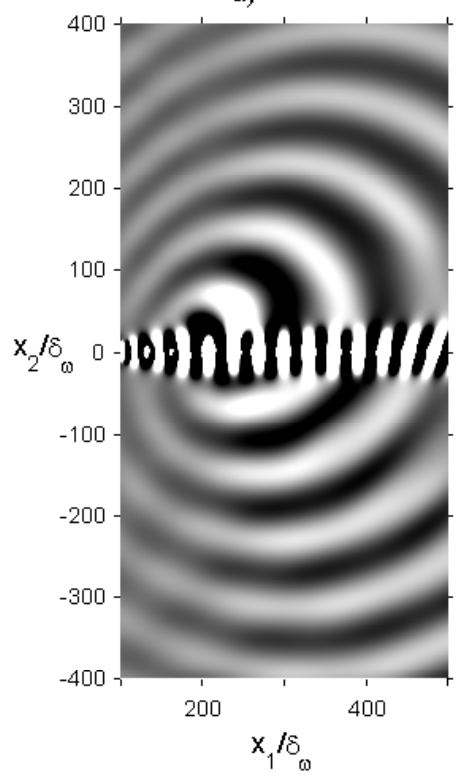

b)

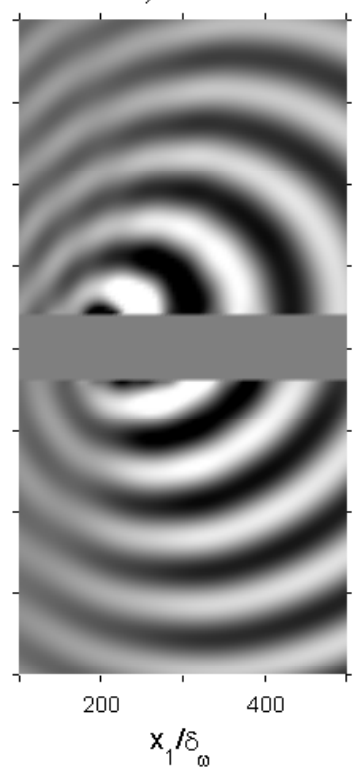

c)

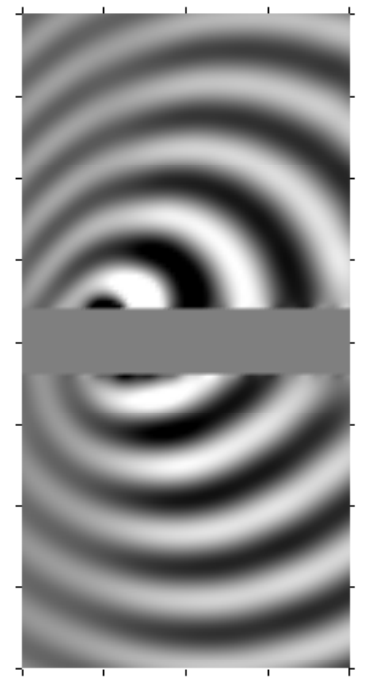

400

200 $x_{1} / \delta$

d)

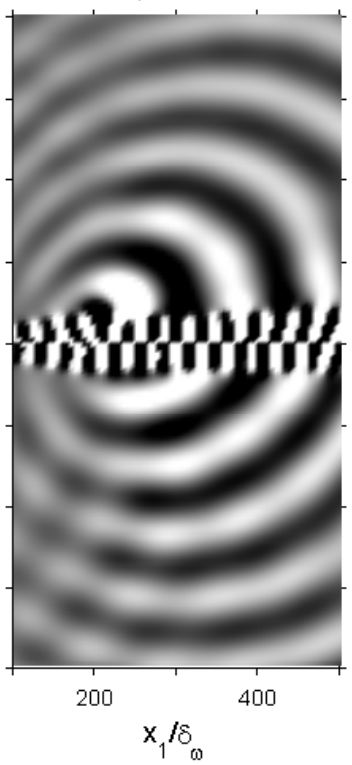

FIG. 2. Instantaneous acoustic pressure field of the mixing layer, predicted by a) DNC b) Lighthill's analogy with convected Green's function (9), c) the convected Kirchhoff method, and d) Lighthill's analogy (6) with the full source domain. Levels are the same for each field, linearly increasing from $-4.0 \times 10^{-5} c_{0}^{2}$ (black) to $4.0 \times 10^{-5} c_{0}^{2}$ (white).

a)

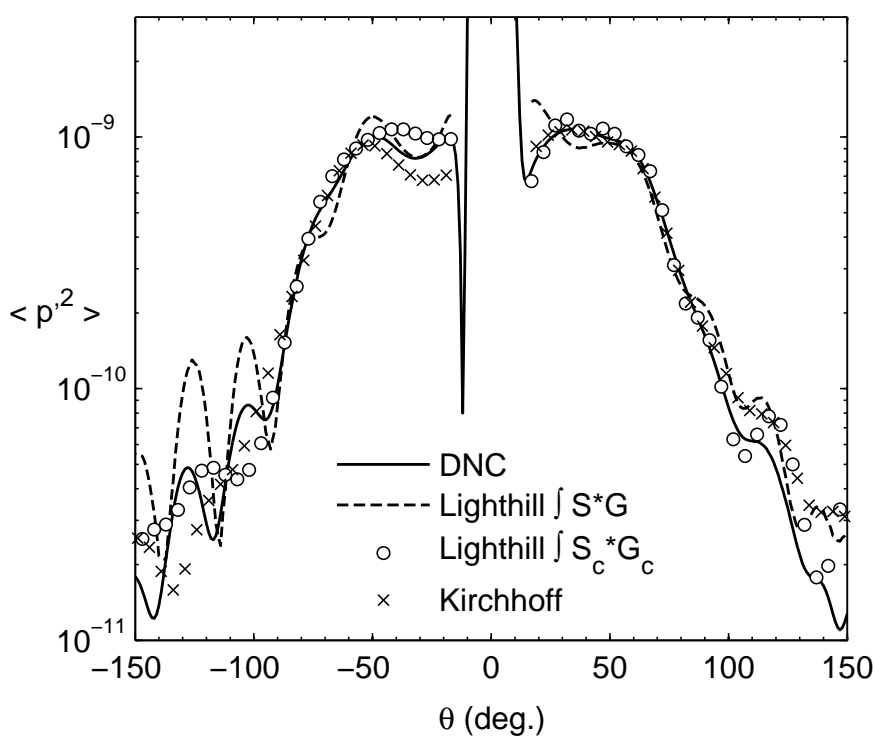

b)

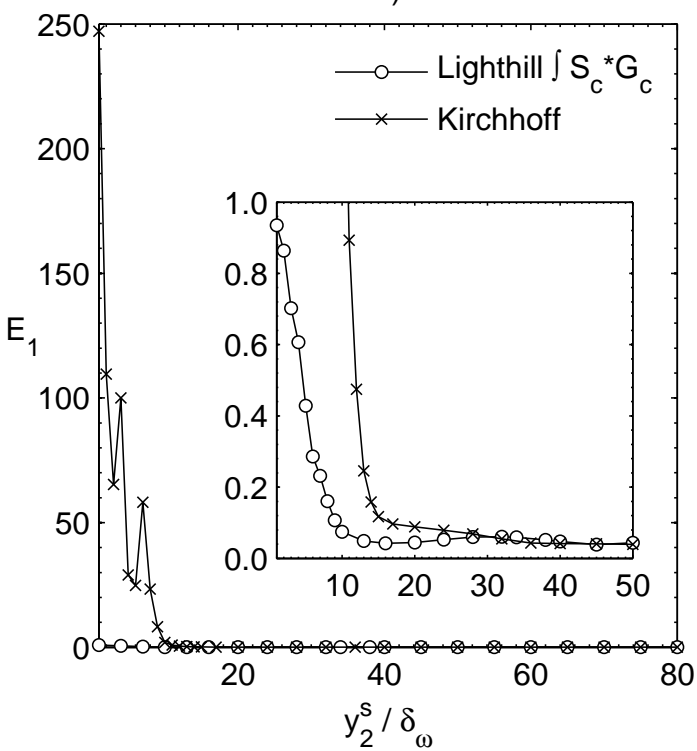

FIG. 3. a) Acoustic intensity at $r=150 \delta_{\omega}$ predicted using 4 approaches. b) Influence of the source domain extent and control surface location.

source term over the whole domain is the most affected by this artefact, especially on the low-speed side.

In figures 2 and $3 \mathrm{a}$ ), the control surface used in the Kirchhoff method is defined by $y_{2}^{s}=40 \delta_{\omega}$, whereas the convected Lighthill source term is integrated over the volume inside this surface. How the predicted acoustic field depends on the control surface position and on the source extent is analysed through figure $3 \mathrm{~b}$ ), where the error with respect to the $\mathrm{DNC}$ is plotted, being defined as:

$$
E_{1}=\frac{\left\|I_{H y b r i d}(\mathbf{x})-I_{D N C}(\mathbf{x})\right\|}{\left\|I_{D N C}(\mathbf{x})\right\|}
$$

where $I$ is the acoustic intensity and $\|f(\mathbf{x})\|$ is the mean value of $|f|$ over a set of observer points. Here, that set is defined as the line $x_{2}=300 \delta_{\omega}$ for $100 \leq x_{1} / \delta_{\omega} \leq 500$. For both hybrid methods, the error converges towards a constant value of about $5 \%$ for $y_{2}^{s}>40 \delta_{\omega}$, but the 
a)

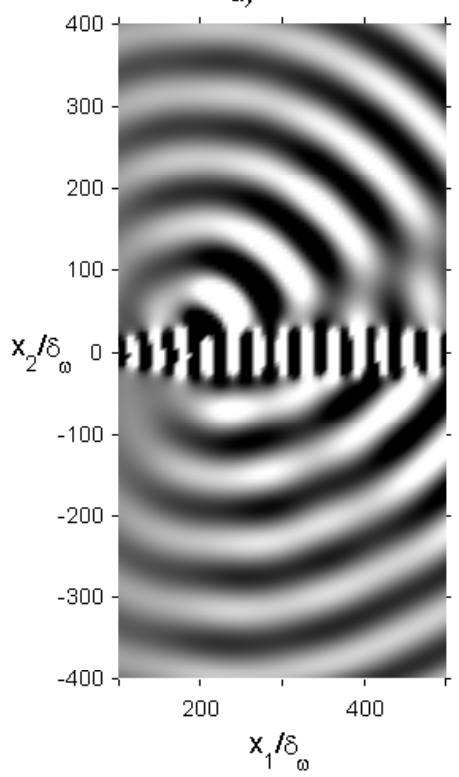

b)

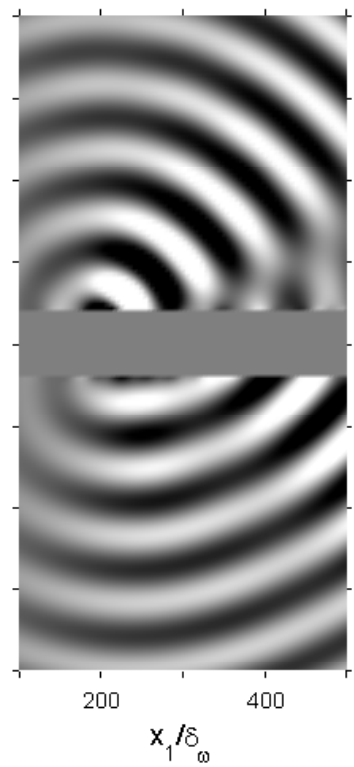

c)

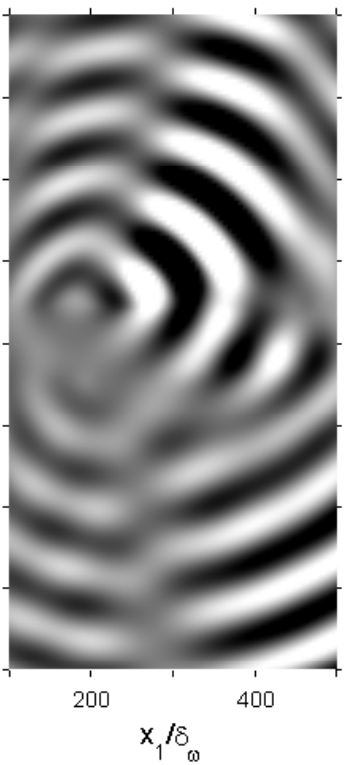

d)

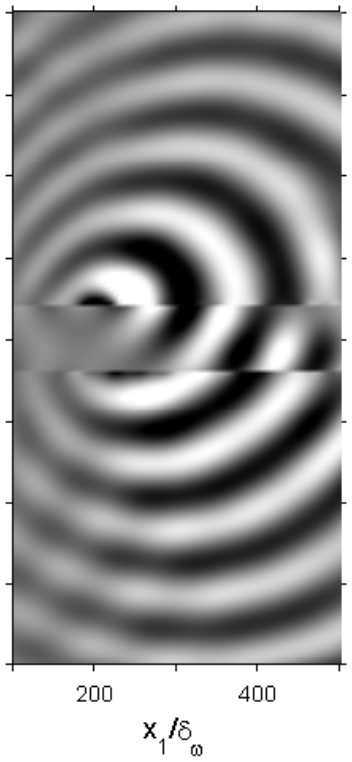

FIG. 4. Hybrid computations with the non-convected Green function. a) Lighthill's analogy (6) integrated over $D_{S O U R C E}$ : $\left|y_{2} / \delta_{\omega}\right|<40 ;$ b) Kirchhoff's method (5) with $M_{\infty}=0$ for $y_{2}^{s}=40 \delta_{\omega}$; c) Lighthill's analogy (6) integrated over $D_{O B S}$ : $40<\left|y_{2} / \delta_{\omega}\right|<400$; and d) sum of b) and c). The sum of a) and c) is identical as figure 2d). Levels are the same for each field, linearly increasing from $-4.0 \times 10^{-5} c_{0}^{2}$ (black) to $4.0 \times 10^{-5} c_{0}^{2}$ (white).

behavior is different for lower values. The convected Lighthill computation reaches the steady error level from $y_{2}^{s} \approx 10 \delta_{\omega}$ after a regular decrease from unity. For the Kirchhoff computation part, the error is much higher when the control surface is located very close to the layer, and it catches the Lighthill curve only for $y_{2}^{s} \gtrsim 30 \delta_{\omega}$.

From this result, the conclusion is that the acoustic source mechanism is confined in $\left|y_{2}\right| \leq 10 \delta_{\omega}$, and that below $y_{2} \approx 30 \delta_{\omega}$, the fluctuations associated with the vortex dynamics dominate in the pressure field and prevent the Kirchhoff integral from a correct extrapolation of the acoustic waves.

\section{B. Uniform flow effect}

A decomposition of the integration domain $D$ is now introduced, in order to analyse convection effect inclusion in the Lighthill source term from a spatial point of view. Using the notations presented in figure 1 , the region defined by $\left|y_{2}\right|<y_{2}^{S}$ is noted $D_{S O U R C E}$ and hereafter called 'the source region', while its complement with respect to $D$ is noted $D_{O B S}$ and called 'the observation region'. Hybrid computations are then performed using the Green function for a propagation medium at rest, that is using (5) with $M_{\infty}=0$ for the Kirchhoff method, and (6) with an integration over $D_{S O U R C E}$ for the Lighthill analogy.

The resulting instantaneous acoustic pressure fields for $y_{2}^{s}=40 \delta_{\omega}$, plotted in figure $4 \mathrm{a}$ ) and $4 \mathrm{~b}$ ), are very similar between the two methods, but the prediction does not agree with the DNC reference shown in figure $2 \mathrm{a}$ ). However, if the contribution of the, still not convected,
Lighthill source term with an integration over $D_{O B S}$, shown in figure 4c), is added to both, the correct pattern is recovered in figure $4 \mathrm{~d}$ ) for the Kirchhoff method, and is the same as in figure $2 \mathrm{~d}$ ) for the Lighthill analogy. The lobes for $|\theta| \geq 90^{\circ}$ are again visible in figure $4 \mathrm{~d}$ ), suggesting they are an effect from the acoustic/propagation domain (ie: from the boundary conditions in the DNC), and not from the source mechanism, otherwise they should have been present in the convected Kirchhoff prediction in figure 2c).

The convection effect can thus be provided to the wave extrapolation from a Kirchhoff surface by the integration of the Lighthill source term over the observer domain, which plays here exactly the same role as the convected Green function, that is, schematically:

$$
\begin{array}{r}
\int_{\left|y_{2}\right|=y_{2}^{s}}\left\{G_{c} \frac{\partial p^{\prime}}{\partial \mathbf{n}}-p^{\prime} \frac{\partial G_{c}}{\partial \mathbf{n}}\right\} \mathrm{d} y_{1} \approx \\
\int_{\left|y_{2}\right|=y_{2}^{s}}\left\{G \frac{\partial p^{\prime}}{\partial \mathbf{n}}-p^{\prime} \frac{\partial G}{\partial \mathbf{n}}\right\} \mathrm{d} y_{1}+\iint_{\left|y_{2}\right|>y_{2}^{s}} S \cdot G \mathrm{~d} \mathbf{y}
\end{array}
$$

Note that the Kirchhoff formalism leads to the same flow quantity in the integrand, namely the pressure and its normal derivative to the control surface, for both the convected and static medium wave equations, unlike the Lighthill formalism, for which the source term expression is dependent on the specific choice of the propagation operator ${ }^{3}$. The fully compressible solution was needed for that illustration, which may not improve the efficiency of hybrid methods, though it confirms the robustness of the interpretation of Lighthill's equation as an implicit equation for (aero)acoustic propagation within a uniformly moving flow. Moreover it is of theoretical in- 
terest that, within the integration process, the convected action of the Ligthill source term is spatially limited to the observer region (provided that the Kirchhoff surface accounts for the whole source process).

Now that the hybrid approaches have been validated when the source quantity is evaluated from the DNC, whether the density variations should be included in the Lighthill source term to ensure a correct prediction is investigated in the following.

\section{DENSITY VARIATIONS IN THE QUADRUPOLES}

Attempting to separate a purely radiating part from flow induced effects, Cabana et al. ${ }^{13}$ suggested a decomposition of the Lighthill source term, featuring density gradient, dilatation and vorticity from the double divergence. Subterms including the two formers were identified as a compressible reaction to subterms identified as driving terms based on vorticity and kinetic energy. Margnat \& Fortuné ${ }^{20}$ applied that decomposition to the spatially evolving mixing-layer and noticed the importance of the dilatation transport term to account for the mean flow effect. Moreover, applying the Reynolds decomposition to density and velocity components of the Lighthill tensor, Moser et al. ${ }^{14}$ reported a significant effect of the term made up with density fluctuations and mean streamwise velocity when the Mach number is increased. Those studies pose the question of the preservation of the source and propagation mechanisms included in the Lighthill source term when the density field is submitted to assumptions, as it may occur in both theoretical works and hybrid predictions.

\section{A. Two assumptions about compressibility}

The analogy approach assumes that the flow drives the acoustics with no feedback from the latter, so that Lighthill's equation can be solved explicitely once the source distribution is known. This is theoretically valid for weakly compressible flows, say, low Mach numbers. In the absence of heating, once a low Mach number is assumed, it is tempting to assume a constant density in the evaluation of the source term, thus replacing $\rho$ by $\rho_{0}$ in the source expression, that is:

$$
S \approx \rho_{0} \frac{\partial^{2} u_{i} u_{j}}{\partial y_{i} \partial y_{j}}=S_{0}
$$

Similarly, we define

$$
S_{c, 0}=\rho_{0} \frac{\partial^{2}}{\partial y_{i} \partial y_{j}}\left[\left(u_{i}-U_{i}^{\infty}\right)\left(u_{j}-U_{j}^{\infty}\right)\right]
$$

This assumption is usually made when the source quantity is evaluated from experimental data in flows without significant density variations (subsonic and isothermal flows): the compressible content of the velocity field is preserved, while density gradients are neglected. It is also often assumed in theoretical works, e. g. ${ }^{21}$.

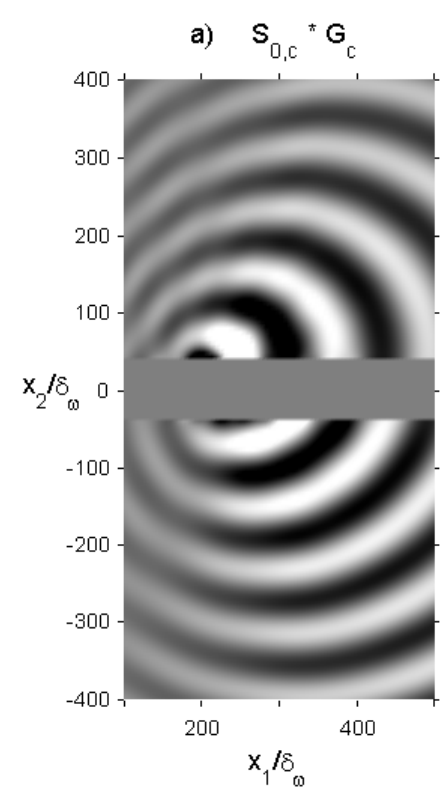

b) $S_{0}{ }^{*} G$ Full domain

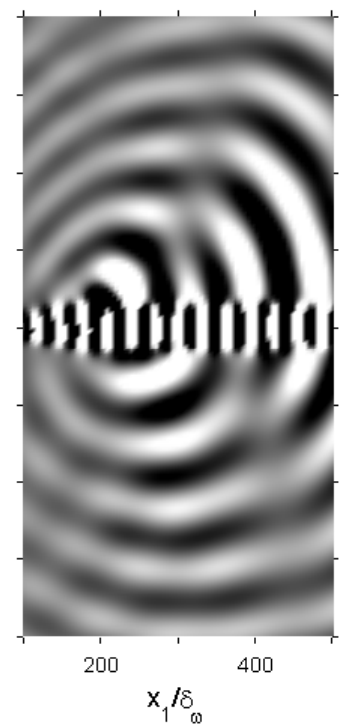

FIG. 5. Hybrid predictions based on Lighthill's analogy with a constant density in source subterms. The acoustic pressure field radiated by a) $S_{c, 0}$ with the convected Green function; b) $S_{0}$ integrated over the full DNC domain. Levels are the same for each field, linearly increasing from $-4.0 \times 10^{-5} c_{0}^{2}$ (black) to $4.0 \times 10^{-5} c_{0}^{2}$ (white). $D_{S O U R C E}$ and $D_{O B S}$ are defined as inside and outside $\left|y_{2}^{s}\right|=40 \delta_{\omega}$ respectively.

For the present mixing layer, figure 5a) shows that neglecting density variations does not alter the acoustic prediction using Lighthill's analogy in the convected form (10). However, for the form (7) that includes the convection effect in the source term, it does, as visible in figure $5 \mathrm{~b}$ ), yielding an incorrect wave pattern.

Note that in acoustic motions, the fluctuating activity involves the pressure and the velocity together with the density, meaning that a constant density assumption will not remove the acoustic part of the Lighthill source term contained in the velocity field. An incompressible simulation, however, will lead to a source quantity evaluated with a divergence-free velocity field, that is:

$$
S_{c} \approx \rho_{0} \frac{\partial^{2}}{\partial y_{i} \partial y_{j}}\left[\left(\hat{u}_{i}-U_{i}^{\infty}\right)\left(\hat{u}_{j}-U_{j}^{\infty}\right)\right]=\hat{S}_{c, 0}
$$

where $\nabla \cdot \hat{\mathbf{u}}=0$. Alternately, $\hat{S}_{0}=\rho_{0} \frac{\partial^{2} \hat{u}_{i} \hat{u}_{j}}{\partial y_{i} \partial y_{j}}$. Such assumption on the source term, which is common in the use of hybrid methods, is investigated in details in the following subsections.

\section{B. Compressible and incompressible near-fields}

For the purpose of evaluating (9) under assumption (17), an incompressible simulation of the mixing layer at the same Reynolds number is conducted. The incompressible flow solver is that presented $i^{22}$. The perturbation of the inflow profile (11) is only applied to the 

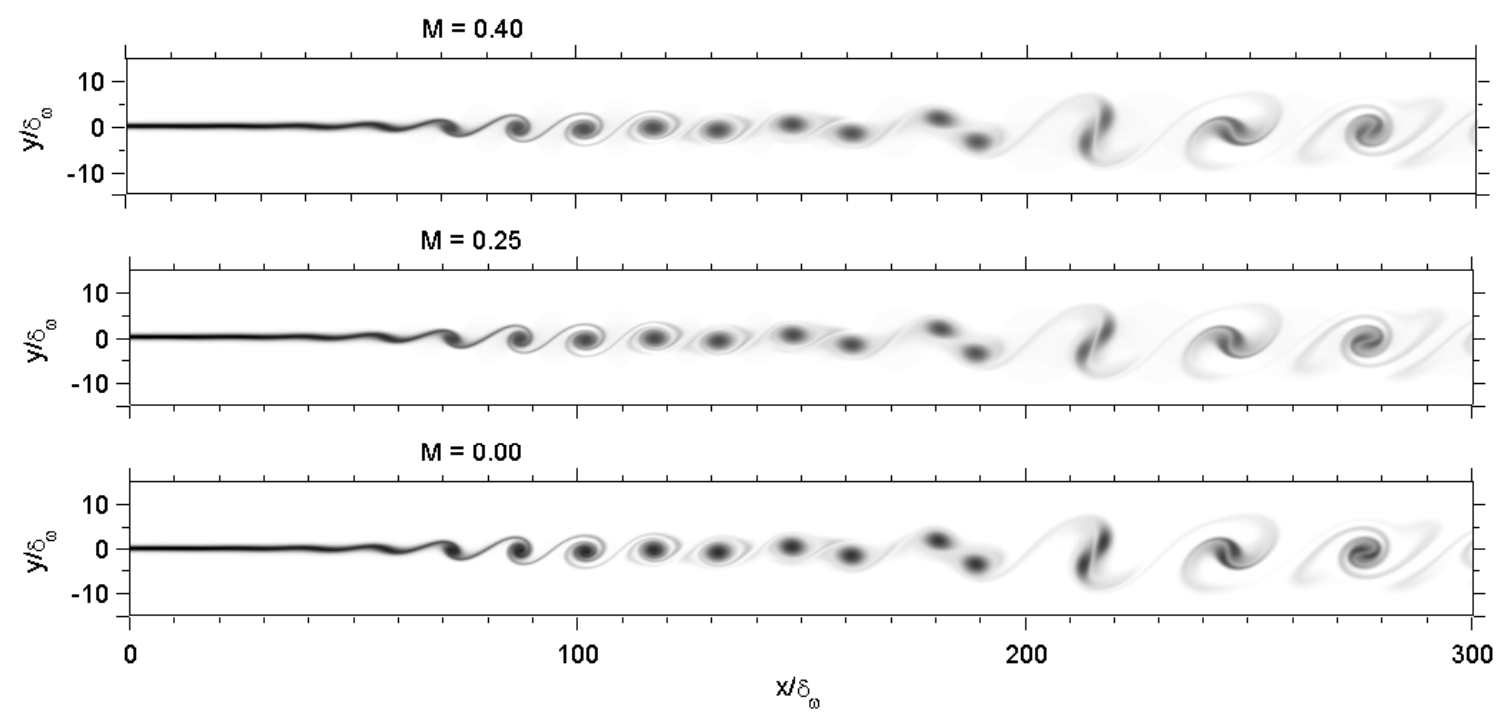

FIG. 6. Vorticity snapshots for DNC's at $M=0.40$ (top) and $M=0.25$ (middle) and for the incompressible simulation (bottom). Levels are from $-0.8 U_{2} / \delta_{\omega}$ (black) to 0.0 (white).

transverse velocity component. The perturbation amplitude at $f_{0}$ is $0.0027 U_{2}$, twice that of $f_{0} / 2$, while the phase between the two frequencies is $0.4 \pi$. These settings are chosen in order to obtain the same longitudinal evolution as obtained in the compressible simulations. The computational domain is limited to $100 \delta_{\omega}$ on each side of the mixing layer since the acoustic field can not be simulated. The dynamic viscosity is linearly increased on the second half of the computational domain, providing a dissipation of paired vortices before the outflow, in order to prepare the treatment of the truncation in the hybrid acoustic prediction.

The resulting vorticity field is compared in figure 6 to that from the DNC at different Mach numbers. As shown by Moser et $a l^{14}$ there are almost no observable differences between the vorticity field of the simulation at $M=0.25$ and that of the simulation at $M=0.40$. No more differences appear when the comparison includes the incompressible simulation. In the following, the velocity and pressure statistics in the mixing region are quantitatively compared between the incompressible simulation and the two compressible cases. The Reynolds decomposition is used noting $f=\bar{f}+f^{\prime}$ where $\bar{f}$ is the mean value of $f$ over a pairing period.

The spreading of the mixing layer is characterised with the help of the streamwise evolution of a thickness. In addition to the vorticity thickness (12), two other definitions are used. How far the constant velocity profiles must be moved to deliver the same mass flow leads to the displacement thickness:

$$
\delta^{*}\left(y_{1}\right)=\int_{-\infty}^{0}\left(\frac{\bar{u}}{U_{2}}-1\right) d y_{2}+\int_{0}^{+\infty}\left(1-\frac{\bar{u}}{U_{1}}\right) d y_{2}
$$

Finally, the summation of the half thickness of each stream yields a third definition:

$$
\delta_{1 / 2}\left(y_{1}\right)=\delta_{1}\left(y_{1}\right)+\delta_{2}\left(y_{1}\right)
$$

where $\delta_{1}$ and $\delta_{2}$ are defined by $\bar{u}\left(y_{1}, \delta_{1}\right)=U_{1}-\Delta U / 4$ and $\bar{u}\left(y_{1}, \delta_{2}\right)=U_{2}+\Delta U / 4$, respectively.

The evolutions of the three thicknesses, of the streamwise and transverse velocity $R M S$ fluctuations and of the instantaneous pressure fluctuation are plotted along the mixing-layer axis in figure 7 . All the quantities show excellent agreement between the three simulations. In particular, the saturation of the first unstable mode occurs at the same position. The vortex pairing causes a global maximum of the thicknesses and the velocity fluctuations, and a second saturation for the pressure fluctuation. The fluctuations from the incompressible simulation have a slower amplitude decrease at the end of the plotted domain, likely because the dissipation region is not designed in the same way. The vorticity thickness exhibits spurious local variations dowstream of the pairing region. Indeed, because it is defined using the maximum slope of the mean velocity profile, its computation may be affected by the presence of more than one slope maximum, which happens after the pairing.

Concerning the transverse decays of fluctuations, it is shown through figure 8 that for $\left|y_{2}\right|<25 \delta_{\omega}$, the fluctuations of both velocity components and of the pressure, once normalized by the low-speed flow velocity, have exactly the same level for the three simulations. That region may then be referred to as hydrodynamic. Above $\left|y_{2}\right|=25 \delta_{\omega}$, the fluctuations from the incompressible simulation go on decaying exponentially, while the acoustic fluctuations start to be dominant in the compressible simulations. The latter depend on the Mach number, since they scale with $M^{7 / 2}$ in $2 \mathrm{D}$ as observed in ref. ${ }^{14}$.

At this point, it is clear from figures 6, 7, and 8, that the hydrodynamic part of the flow is the same between both compressible simulations and the incompressible one. On that basis, the assumption of an incompressible velocity field can be tested in the Lighthill prediction. 


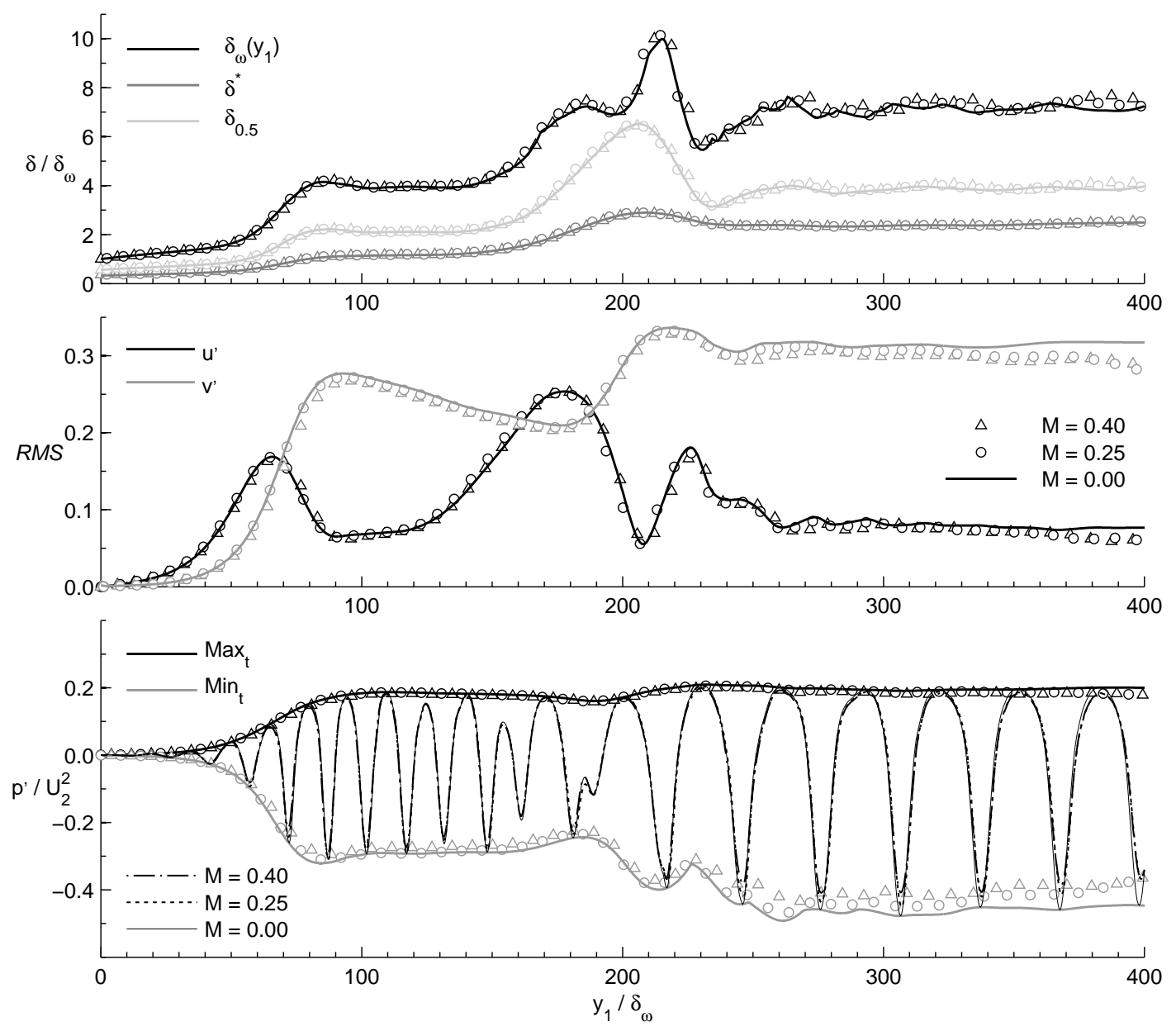

FIG. 7. Evolution of statistic quantities along the mixing-layer axis. Top: mixing-layer thicknesses; middle: fluctuating longitudinal and transverse velocities; bottom: instantaneous pressure fluctuation and its extreme values in time. $M=0.00$ stands for the incompressible simulation.

\section{Results for the convected Lighthill predictions}

In figure 9, the directivity of the acoustic intensity at $r=150 \delta_{\omega}$ obtained using either $S_{c, 0}$ or $\hat{S}_{c, 0}$ is compared to the convected Lighthill computation using the fully compressible source term $S_{c}$. The DNC result is shown too, for reference. Three extents of the source region are considered, in order to visualise any phenomenon that could be missed where the hydrodynamic and acoustic parts coexist. A first worthnoting result appears through the intensity fall for $|\theta|>50^{\circ}$, which is well captured by all the Lighthill predictions. Below these angles, the two sides of the mixing layer do not exhibit the same trends. On the high-speed side, the modelling of the source term does not significantly affect the prediction, even for the smallest source extent. The incompressible source term tends to yield a slight overprediction at small $|\theta|$, however. On the low-speed side, a strong overprediction at aft angles is observed for the three hybrid computations when the source region is limited to $\left|y_{2}\right| \leq 20 \delta_{\omega}$. For larger source extents, the constant density modelling yields nearly the same result as the fully compressible source term, while the incompressible assumption still leads to an overprediction at small $|\theta|$, though reduced.

That influence of the source extent suggests a weakly compressible phenomenon in the near-field, which affects the acoustic radiation while being accounted for by the Lighthill source term if evaluated with a compressible velocity field. Regarding this, the pressure fluctuation near-field is plotted in figure 10 for both flow simulations. The DNC makes visible a pattern of two lines of spots where the fluctuation level is strongly lower than around, like sinks, downstream after the pairing and around $y_{2}=$ $\pm 30 \delta_{\omega}$. The incompressible flow simulation returns only one such sink, in the vicinity of the pairing. Moreover, an asymmetry between the two streams is noticed, the high-speed flow exhibiting more similarities between the two simulations. Such near-fields may influence strongly the propagation at small angles (for which acoustic rays stay longer in the perturbed region), what could explain why any of the convected Lighthill computations miss the acoustic level there when the source domain do not include it, and why the incompressible source modelling misses this in the low-speed region even for a larger source domain. 

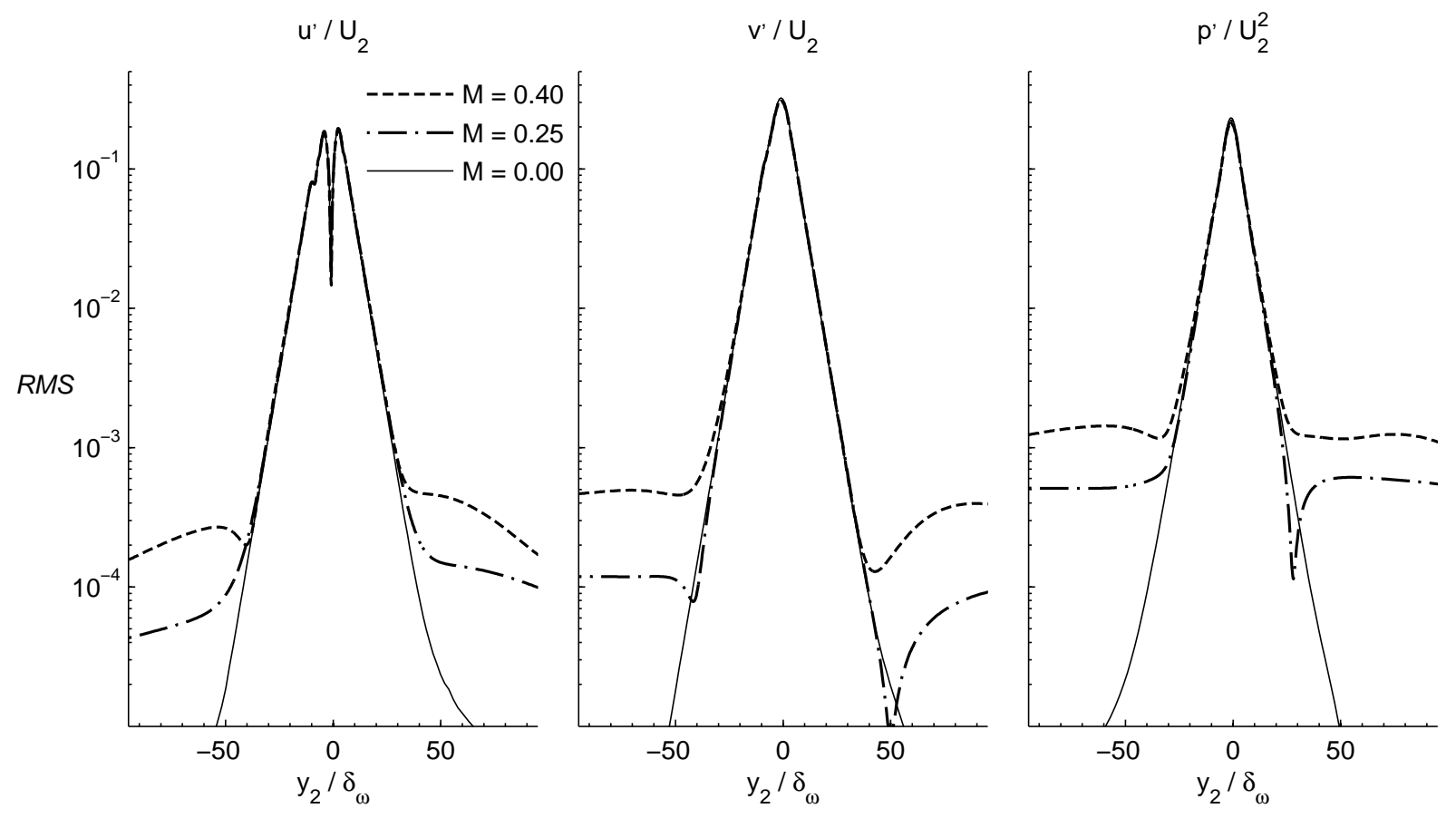

FIG. 8. Transverse evolution of the velocity and pressure fluctuations at $x / \delta_{\omega}=300 . M=0.00$ stands for the incompressible simulation.

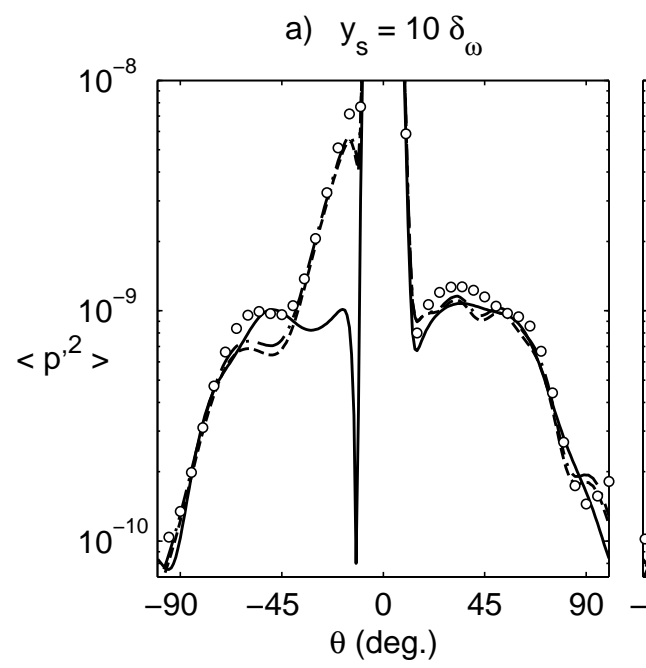

b) $y_{s}=20 \delta_{\omega}$

c) $\mathrm{y}_{\mathrm{s}}=40 \delta_{\omega}$
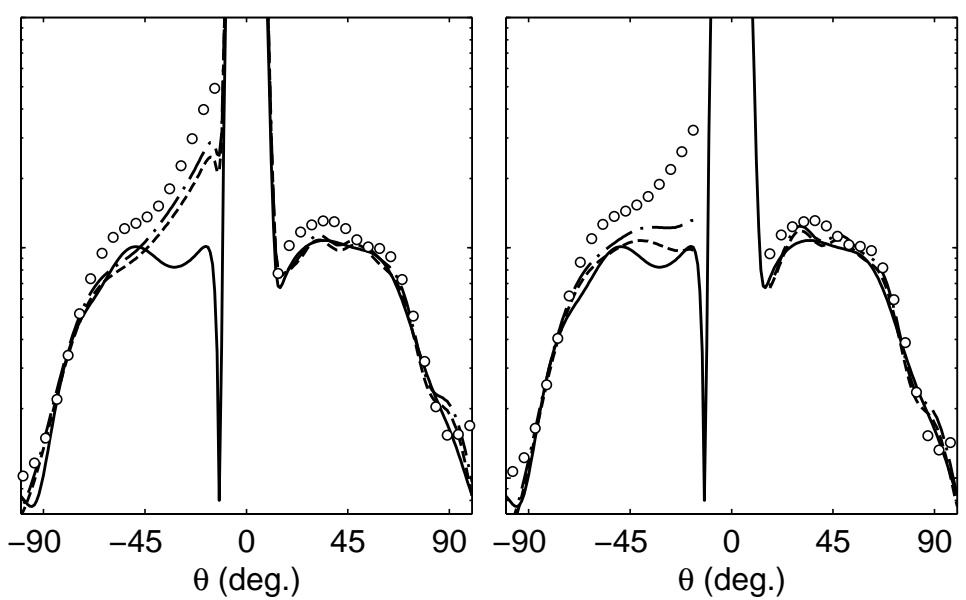

FIG. 9. Influence of the source modelling on the acoustic directivity at $r=150 \delta_{\omega}$ predicted by the convected Lighthill analogy with the fully compressible source term $S_{c}$ (dashed line), a constant density assumption $S_{c, 0}$ (dash-dotted line), and incompressible velocity fields $\hat{S}_{c, 0}$ (symbols). Full line: DNC. Three extensions of the source domain, case $M=0.25$.

\section{Increasing the Mach number}

As investigated by Moser et $a l^{14}$, increasing the Mach number of mixing layers with $U_{1}=2 U_{2}$ leads to a reinforcement of the directivity peak around $\theta=50^{\circ}$ especially for the radiation towards the high-speed flow. This is illustrated in figure 11a) and figure 11c) with the instantaneous acoustic pressure field as given by the DNC for $M=0.25$ and $M=0.4$ respectively. That reinforcement is perfectly captured by the incompress- ible source term modelling, as qualitatively visible in figure 11b) and figure 11d), and quantitavely confirmed by the intensity plots at $r=150 \delta_{\omega}$ in figure 12 showing well the intensity fall after $\theta \approx 50^{\circ}$. In spite of the aforementioned flaw at aft angles, such a successful association of the incompressible source modelling with the convected Green function, even for the highly subsonic case at $\left(M_{1}=0.8, M_{2}=0.4\right)$, is worth to notice.

Furthermore, it can be deduced that the effect of the Mach number on the directivity is brought by the acous- 

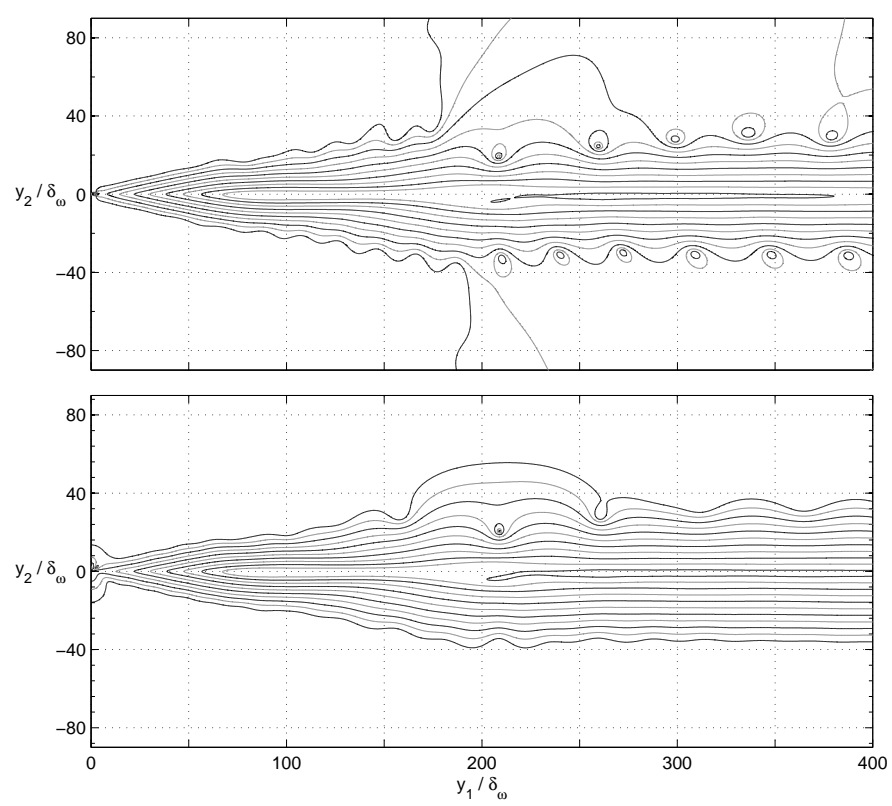

FIG. 10. Isocontours of the $R M S$ pressure fluctuation in the near-field of the compressible (top) and incompressible (bottom) mixing layer, for $M=0.25$. The level of a contour is twice the previous one, starting from $0.0032 U_{2}^{2}$.

tic wave convection only, because the three vortical fields do not show any significant difference while the convolution to the convected Green function yields that directivity evolution. This is consistent with Ffowcs Williams' theoretical work ${ }^{23}$, according to which there may be no refraction effect of shear at the interface between two uniform flows of different speed and density, in the limit of a small wavelength with respect to the transverse extent of the flow and large with respect to the interface thickness. The latter conditions are satisfied in the present mixing layers, where the wavelength is about $50-80 \delta_{\omega}$ and the transverse extent is indeed infinite due to the boundary conditions (in any case, the computational domain is $400 \delta_{\omega}$ on each side of the mixing-layer).

\section{CONCLUDING REMARKS}

Based on several expressions of the source term, comprehensive hybrid predictions were carried out in order to bring facts about which content of the Lighthill source quantity should be taken into account, regarding the inclusion of density fluctuations and the flow effects on acoustics. The mixing-layer flow case was selected in order to focus on free shear flows while avoiding wall effects. This apparently simple 2D case still requires careful numerical implemention. Instantaneous pressure fields were preferred to point spectra in order to visualise wavefront patterns.

Strong background is provided about the modelling of the quadrupole term, the principal conclusions being:

- The constant density assumption is numerically validated.
- The use of incompressible velocity fields in Lighthill source term is numerically validated, except for the radiation at low angles.

- The validity of both assumptions is limited, however, to the source region, because they cancel out the inclusion of convection effects in the Lighthill source term. Those effects are correctly predicted when the source quantity is fully compressible (e. g. from DNC) and when a convected Green function is associated to either constant density or incompressible quadrupoles.

The two following peripheral points are also emphasized:

- While the hydrodynamic pressure field from the incompressible simulation matches perfectly with those from the DNC, it yields an incorrect acoustic prediction when put into the Kirchhoff formalism. This is an expected result when the control surface is far from the vortical region, but it shows that the pressure field generated by the hydrodynamic waves is not appropriate as such to model the aeroacoustic excitation by the (2D, low-Re) mixing-layer.

- Finally, because the reinforcement of the directivity around $|\theta| \approx 50^{\circ}$ due to the Mach number increase is well captured with incompressible sources and a convected Green function, it is concluded that such effect of the Mach number is not refraction in the sheared region but convection in the observer region.

\section{Acknowledgments}

The authors gratefully acknowledge Eric Lamballais and Veronique Fortuné for courtesy share of the flow solvers and compressible data.

${ }^{1}$ M. J. Lighthill, "On sound generated aerodynamically. I. general theory", Proc. Roy. Soc. A 223, 1-32 (1952).

2 S. C. Crow, "Aerodynamic sound emission as a singular perturbation problem", Stud. Appl. Math. 49, 1-44 (1970).

3 M. E. Goldstein, "On identifying the true sources of aerodynamic sound", J. Fluid Mech. 526, 337-347 (2005).

${ }^{4}$ G. M. Lilley, "On the noise from jets", Technical Report CP-131, AGARD (1974).

${ }^{5}$ H. S. Ribner, "Effects of jet flow on jet noise via an extension to the Lighthill model", J. Fluid Mech. 321, 1-24 (1996).

6 T. Colonius, S. K. Lele, and P. Moin, "Sound generation in a mixing-layer", J. Fluid Mech. 330, 375-409 (1997).

7 T. Suzuki and S. K. Lele, "Green's functions for a source in a mixing layer: direct waves, refracted arrival waves and instability waves", J. Fluid Mech. 477, 89-128 (2003).

8 A. Samanta, J. B. Freund, M. Wei, and S. K. Lele, "Robustness of acoustic analogies for predicting mixing-layer noise", AIAA Journal 44, 2780-2786 (2006).

9 M. E. Goldstein, Aeroacoustics (McGraw-Hill Book Co.), 293 p. (1976). 
a)

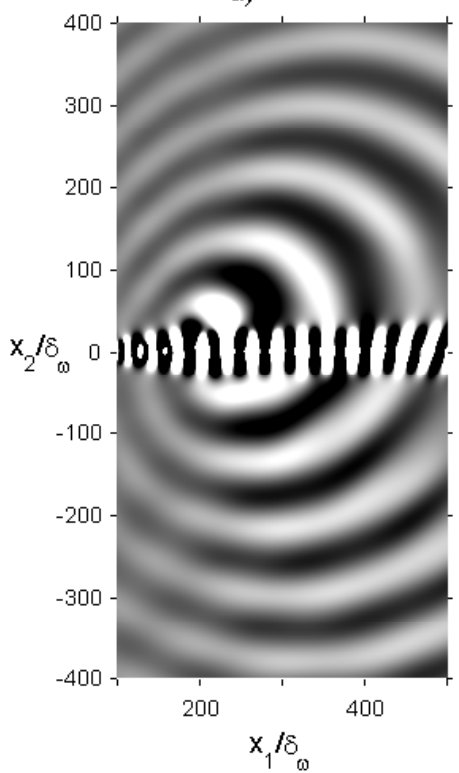

b)

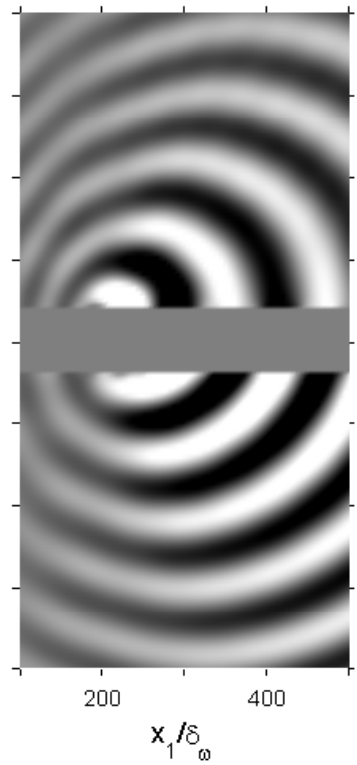

c)

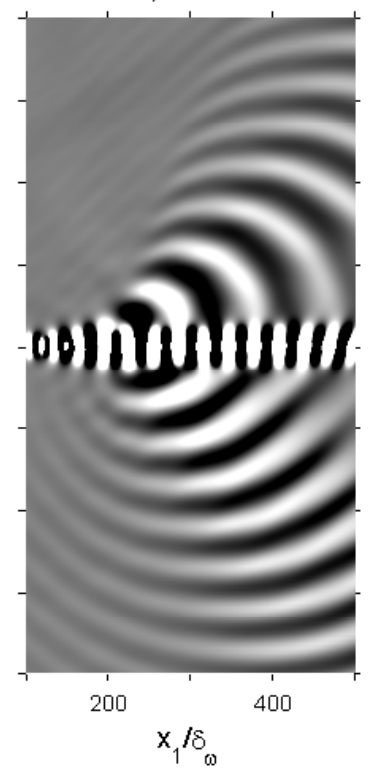

d)

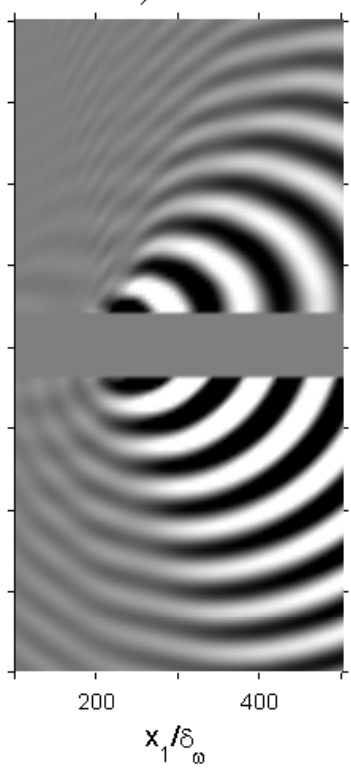

FIG. 11. Instantaneous acoustic pressure field of the mixing layer predicted by DNC (a) and c)) and convected Lighthill's analogy with incompressible source data and $y_{2}^{s}=40 \delta_{\omega}$ (b) and d)), for $M=0.25$ (a) and b), levels from $-4.0 \times 10^{-5} c_{0}^{2}$ (black) to $4.0 \times 10^{-5} c_{0}^{2}$ (white) ) and $M=0.4(\mathrm{c})$ and d), levels are five times larger).

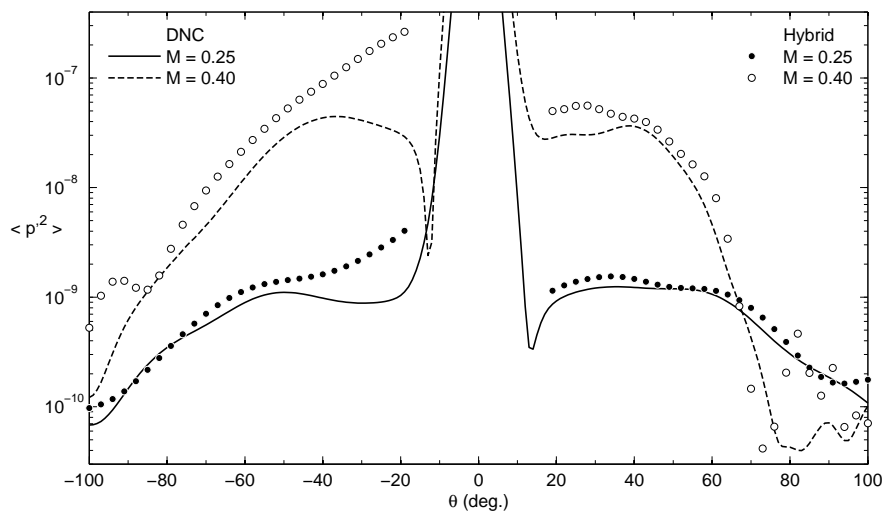

FIG. 12. Acoustic intensity at $r=150 \delta_{\omega}$ predicted by DNC and convected Lighthill's analogy with incompressible source data and $y_{2}^{s}=40 \delta_{\omega}$, for two Mach numbers.

10 D. P. Lockard, "An efficient, two dimensional implementation of the Ffowcs Williams and Hawkings equation", J. Sound Vib. 229, 897-911 (2000).

11 X. Gloerfelt, C. Bailly, and D. Juvé, "Direct computation of the noise radiated by a subsonic cavity flow and application of integral methods", J. Sound Vib. 266, 119-146 (2003).

12 C. L. Morfey, C. J. Powles, and M. C. M. Wright, "Green's functions in computational aeroacoustics", Int. J. Aeroacoutics 10, 117-160 (2011).

13 M. Cabana, V. Fortuné, and P. Jordan, "Identifying the radiating core of Lighthill's source term", Theoret. Comput. Fluid Dynamics 22, 87-106 (2008).

14 C. Moser, E. Lamballais, F. Margnat, V. Fortuné, and Y. Gervais, "Numerical study of Mach number and ther- mal effects on sound radiation by a mixing layer", Int. J. Aeroacoutics 11, 555-580 (2012).

15 D. E. Amos, "A portable package for bessel functions of a complex argument and nonnegative order", ACM Trans. Math. Software 12, 265-273 (1986).

16 P. Martínez, A. Pradera, and C. Schram, "Efficient implementation of equivalent sources from CFD data in curle's analogy.", AIAA paper 2007-3569 (2007).

17 D. Obrist and L. Kleiser, "The influence of spatial domain truncation on the prediction of acoustic far-fields", AIAA paper 2007-3725 (2007).

18 C. Schram, "A boundary element extension of curle's analogy for non-compact geometries at low-mach numbers", J. Sound Vib. 322, 264-281 (2009).

19 C. Bogey, X. Gloerfelt, and C. Bailly, "An illustration of the inclusion of sound-flow interactions in Lighthill's equation", AIAA Journal 41, 1604-1606 (2003).

${ }^{20}$ F. Margnat and V. Fortuné, "An iterative algorithm for computing aeroacoustic integrals with application to the analysis of free shear flow noise", J. Acoust. Soc. Am. 128, 1656-1667 (2010).

${ }^{21}$ P. Huerre and D. G. Crighton, "Sound generation by instability waves in a low Mach number jet.", AIAA paper 83-0661 (1983).

22 S. Laizet and E. Lamballais, "High-order compact schemes for incompressible flows: A simple and efficient method with quasi-spectral accuracy", J. Comp. Phys. 228, 59896015 (2009).

23 J. E. Ffowcs Williams, "Sound production at the edge of a steady flow", J. Fluid Mech. 66, 791-816 (1974). 